\title{
CONCORDANCIA EN LA EVALUACIÓN DEL DESARROLLO PUBERAL MEDIANTE LA ESCALA DE TANNER ENTRE ADOLESCENTES Y UN MÉDICO ENTRENADO
}

\author{
Sandra Milena Rueda-Quijano ${ }^{1, a}$, Mónica Andrea Amador-Ariza ${ }^{1, b}$, Ana María Arboleda ${ }^{1, c}, J^{\prime}$ hanna Otero ${ }^{2, \mathrm{~d}}$, \\ Daniel Cohen ${ }^{1,2, e}$, Paul Anthony Camacho ${ }^{1,3, f}$, Patricio López Jaramillo ${ }^{1,2,9}$
}

\begin{abstract}
RESUMEN
Objetivos. Evaluar la concordancia entre la autoevaluación del desarrollo puberal mediante la escala de Tanner realizada por adolescentes, comparado con la evaluación efectuada por un médico entrenado. Materiales y métodos. Los datos de 244 adolescentes fueron recolectados como parte del proyecto SIMAC. En la línea de base incluimos una evaluación antropométrica y del desarrollo puberal, con una autoevaluación por parte de los participantes. Resultados. Incluimos 229 adolescentes de 12 a 17 años. La concordancia entre la autoevaluación y la evaluación clínica de la escala de Tanner presentó un acuerdo obtenido de $88,3 \%$ y un coeficiente kappa ponderado de 0,554 . La autoevaluación en mujeres demostró una concordancia buena ( $k a p p a_{\text {pond }}=0,653$ ), y en varones una concordancia moderada $\left(\mathrm{kappa}_{\mathrm{pond}}=0,464\right)$. La única variable con una influencia significativa sobre la capacidad de acuerdo fue el sexo; la probabilidad de desacuerdo en los varones fue $63 \%$ mayor que en las mujeres, independiente de edad, talla y peso (IC 95\%:1,18-2,26). Conclusiones. La autoevaluación no fue lo suficientemente precisa para reemplazar el examen médico, sobre todo en los varones. Se requieren más estudios en relación con este tema, reconociendo el impacto que podría llegar a tener la autoevaluación del desarrollo sexual.
\end{abstract}

Palabras clave: Crecimiento y desarrollo; Adolescente; Autoreporte; Desarrollo del niño; Pubertad (fuente: DeCS BIREME).

\section{CONCORDANCE OF THE ASSESSMENT OF PUBERTAL DEVELOPMENT WITH THE TANNER SCALE BETWEEN ADOLESCENTS AND A TRAINED PHYSICIAN}

\begin{abstract}
Objectives. To assess the concordance between self-assessment of pubertal development with the Tanner scale performed by adolescents compared to the assessment performed by a trained physician. Materials and Methods. As part of the SIMAC project, data was collected on 244 adolescents. At baseline, we included an anthropometric and pubertal development assessment, with a self-assessment by the participants. Results. We included 229 teenagers from 12 to 17 years old. The agreement between the self-assessment and the clinical evaluation with the Tanner scale presented an $88.3 \%$ agreement and a weighted kappa coefficient of 0.554 . The self-assessment in women showed a good agreement ( kappaweighted $=0.653$ ) and in men a moderate agreement (kappaweighted $=0.464$ ). The only variable with a significant influence on the agreement ability was gender; the disagreement probability in males was $63 \%$ greater than in females, regardless of age, height, or weight $(95 \% \mathrm{Cl}: 1,18-2,26)$. Conclusions. Self-assessment was not accurate enough to replace medical examination, especially in males. More research is needed on this subject considering the impact that self-assessment of sexual development could have.
\end{abstract}

Keywords: Growth and development; Adolescent; Self report; Child development; Puberty (source: MeSH NLM).

\footnotetext{
Dirección de Investigaciones, Fundación Oftalmológica de Santander (FOSCAL). Santander, Colombia Universidad de Santander (UDES). Santander, Colombia

Facultad de Medicina, Universidad Autónoma de Bucaramanga (UNAB). Bucaramanga, Colombia

Médico; ${ }^{\mathrm{b}}$ fisioterapeuta; ${ }^{\mathrm{c}}$ microbióloga; ${ }^{\mathrm{d}}$ odontóloga, magister en Salud Pública; ${ }^{\mathrm{e}} \mathrm{PhD}$ en Ciencias del Deporte; ${ }^{\mathrm{f}}$ médico, magister en Epidemiología; ${ }^{\mathrm{g}}$ médico endocrinólogo, $\mathrm{PhD}$ en Ciencias Biológicas

Recibido: 11/12/2018 Aprobado: 21/08/2019 En línea: 19/09/2019
}

Citar como: Rueda-Quijano SM, Amador-Ariza MA, Arboleda AM, Otero J, Cohen D, Camacho PA, et al. Concordancia de la evaluación del desarrollo puberal mediante la escala de Tanner entre adolescentes y un médico entrenado. Rev Peru Med Exp Salud Pública. 2019;36(3):408-13. doi: http://dx.doi.org/10.17843/rpmesp.2019.363.4099. 


\section{INTRODUCCIÓN}

La determinación del estado de desarrollo puberal es un elemento importante del examen físico en niños y adolescentes durante la evaluación del estado de salud (1). El término pubertad es utilizado para definir la transición de un estadio de inmadurez sexual a un estadio de madurez sexual ${ }^{(2)}$. En esta transición existe cierta variabilidad en tiempo, secuencia y ritmo, sin embargo, está conformada por una serie de eventos que generalmente presentan un patrón predecible, por lo que pueden ser usados dentro de la evaluación clínica ${ }^{(2)}$.

La secuencia de eventos del desarrollo puberal difiere según el sexo. En mujeres, la primera característica sexual secundaria detectable en el examen físico es la telarquia, que hace referencia al desarrollo de los senos, y en un $15 \%$ aparece el vello púbico, o pubarquia, como manifestación inicial ${ }^{(3)}$. En varones, la primera característica sexual secundaria es el aumento del volumen testicular, el cual ocurre aproximadamente seis meses antes del crecimiento del pene y la pubarquia ${ }^{(3,4)}$.

El sistema de estadificación utilizado con mayor frecuencia es la clasificación de madurez sexual, también conocido como etapas de Tanner ${ }^{(2)}$, descritas y evaluadas por Tanner y Marshall ${ }^{(5-7)}$, las cuales detectan las primeras características sexuales secundarias al examen físico: telarquia, pubarquia y crecimiento testicular. Este método compara el desarrollo de los caracteres sexuales secundarios del paciente mediante fotografías estandarizadas que van entre el grado 1 (ausencia de desarrollo o estado prepuberal) hasta el grado 5 (desarrollo completo), utilizando únicamente la inspección del examen físico como herramienta. Si se realiza palpación del tejido mamario y medición del volumen testicular, la estimación del estadio de desarrollo puberal mejora ${ }^{(8)}$.

El rango normal de inicio de la pubertad abarca entre cuatro a cinco años, esta amplitud hace que sea difícil diferenciar un desarrollo sexual normal de uno anormal. Por lo tanto, es fundamental que los límites de edad para un desarrollo puberal normal sean actualizados periódicamente y de forma independiente por cada región. En Colombia, la Sociedad Colombiana de Pediatría (SCP) a través de su Programa de educación continua en Pediatría (Precop) (https://scp.com.co) ha generado algunas directrices en torno a la evaluación del desarrollo sexual. Por ejemplo, en varones, un testículo de $3 \mathrm{cc}$ de volumen o una longitud de $2,2 \mathrm{~cm}$ o un vello púbico en la base del pene indican el inicio del desarrollo sexual (Tanner 2). En mujeres, está determinado por las glándulas mamarias, el vello púbico o por la aparición de la menarquia.

A pesar de no haberse establecido una medida de edad para el crecimiento de los senos, testículos o del vello púbico, el Precop de la SCP define desarrollo puberal precoz en las mujeres con un límite de edad entre 8 a 9,5

\section{MENSAJES CLAVE}

Motivación para realizar el estudio. La evaluación del desarrollo puberal es parte del abordaje integral del adolescente, dado las influencias que tiene sobre el desarrollo biológico, social y psicológico. Es importante conocer el grado de introspección de los adolescentes frente a su desarrollo sexual.

Principales hallazgos. El acuerdo obtenido entre los adolescentes y el médico fue del $88,3 \%$; sin embargo, el índice kappa ponderado fue moderado. Las mujeres tuvieron una mejor concordancia interobservador con el médico que los varones.

Implicancias. Es importante fortalecer el conocimiento sobre los procesos de crecimiento, facilitando la identificación y detección temprana de alteraciones del desarrollo.

años, dependiendo de la característica evaluada, y en varones con una edad de 9 años; lo que podría generar una idea de una edad promedio ${ }^{(9)}$. Sin embargo, la autoevaluación del desarrollo sexual por el propio menor, con base de fotografías, es una alternativa válida ${ }^{(10,11)}$.

Evaluar de forma regular el desarrollo sexual es parte del abordaje integral en pediatría, teniendo en cuenta la asociación establecida entre la edad biológica y cronológica y lo que representa para el estado de salud de un niño o un adolescente. Adicionalmente, complementa el abordaje de un diagnóstico nutricional o pondo-estatural; por ejemplo, la causa más frecuente de retraso puberal en ambos sexos es el retraso constitucional del crecimiento y del desarrollo. La evaluación del desarrollo puberal tiene como desventaja el requerir una evaluación directa, ya sea por inspección o palpación, de las mamas y genitales en las mujeres y los genitales en los varones. Esto lo hace difícil de aplicar en evaluaciones grupales (por ejemplo, colegios, menores institucionalizados, etc.) e incómodo para los pacientes (especialmente si el observador es del sexo opuesto) ${ }^{(7)}$.

Hasta el momento, en Colombia no se han realizado estudios que busquen evaluar la correlación existente entre la autoevaluación del desarrollo puberal y la estadificación a cargo de personal entrenado. El objetivo del presente estudio fue evaluar la concordancia entre métodos de la autoevaluación del desarrollo puberal mediante la escala de Tanner de adolescentes de 12 y 17 años del departamento de Santander, comparado con la evaluación efectuada por un médico entrenado.

\section{MATERIALES Y MÉTODOS}

Se realizó un estudio observacional, descriptivo, de corte transversal de la línea basal del proyecto «Fuerza muscular y capacidad aeróbica relación simbiótica en escolares con bajo peso al nacer y riesgo metabólico» denominado SIMAC. SIMAC es un ensayo clínico controlado aleatorizado, registrado en Clinical Trials NTC03779737 
(https://clinicaltrials.gov/ct2/show/NCT03779737), llevado a cabo entre febrero y diciembre de 2018 en una institución educativa pública del municipio de Piedecuesta en el departamento de Santander, Colombia, que tenía como objetivo evaluar la relación entre peso al nacer, adaptaciones al ejercicio aeróbico y de fuerza muscular, y sus efectos en riesgo metabólico, composición corporal y capacidad física en una población adolescente.

La población objetivo fueron adolescentes de 12 a 17 años, residentes en la ciudad de Bucaramanga y su área metropolitana. El tamaño muestral se calculó para evaluar la diferencia entre el consumo máximo de oxígeno (VO2máx) entre el grupo control y los grupos intervenidos del $20 \%$, empleando un método ANCOVA, con una correlación de 0,5 entre las valoraciones inicial y final, error alfa de 0,05 y un poder de 0,8 ; obteniéndose una muestra de 60 adolescentes para cada grupo de bajo peso al nacer y peso adecuado para la edad gestacional. SIMAC contó con la aprobación del Comité de Ética de Investigación de la Fundación Oftalmológica de Santander (FOSCAL). Previo al inicio del estudio, los padres o acudientes legales y los participantes fueron informados acerca del objetivo y proceder del estudio. Los padres otorgaron consentimiento informado y los adolescentes su asentimiento informado.

Como parte de la medición de la línea de base, se incluyó la evaluación antropométrica (peso y talla), y la evaluación del desarrollo puberal. Los participantes fueron tallados $y$ pesados en bipedestación con un estadiómetro de pared fijo marca SECA ${ }^{\circledast}$ y descalzos en una pesa marca TANITA ${ }^{\circledR}$, respectivamente. El porcentaje de grasa se obtuvo a través de la pesa TANITA ${ }^{\circledR}$. La circunferencia de la cintura se midió a la altura del punto medio entre el último borde costal y la cresta iliaca, y la circunferencia de la cadera se midió a la altura del punto de máxima circunferencia sobre las nalgas con una única cinta métrica. La talla fue codificada en una base de datos en centímetros $(\mathrm{cm})$ y el peso en kilogramos $(\mathrm{Kg})$. Se realizó una medición única y se tomó en cuenta un decimal.

Para la evaluación de la concordancia entre métodos del desarrollo puberal entre los adolescentes y el médico entrenado se tuvo en cuenta dos fases: Primero, un médico entrenado les explicaba a los participantes en qué consistía la autoevaluación y la evaluación clínica de la escala de Tanner con ayuda de fotografías de los cinco estadios por sexo ${ }^{(12,13)}$. Después de resolver dudas, y de tener el asentimiento de los participantes, se les solicitaba autoevaluar su desarrollo puberal según las fotografías estandarizadas. Segundo, cada participante fue evaluado por un médico entrenado quien realizó una inspección del desarrollo mamario en mujeres, genitales en varones y vello púbico en ambos sexos. No fue medido volumen testicular, ni fue palpado el tejido mamario. El médico utilizó imágenes prestablecidas de los cinco estadios de Tanner para clasificar al escolar según su desarrollo sexual alcanzado. Para respetar la privacidad y promover la comodidad, cada participante fue examinado de forma privada en una sala especialmente habilitada. El grado de desarrollo puberal alcanzado se evaluó clasificando a los escolares en alguno de los cinco estadios de Tanner descritos para ambos sexos.

Las características de la población se resumieron mediante frecuencias y porcentajes, y medidas de tendencia central y dispersión según la distribución de frecuencias. La normalidad de las variables continuas se evaluó empleando la prueba de Shapiro Wilk. Se hizo una estimación de las diferencias de las características demográficas y antropométricas por sexo con la prueba de chi cuadrado, prueba de Fischer, t de Student o Mann Whitney según la distribución de frecuencias. La concordancia entre métodos (evaluación clínica y la autoevaluación) se estimó para el acuerdo de la identificación del estadiaje de madurez sexual con el índice de Kappa de Cohen ponderada. La interpretación del índice de Kappa se hizo con los criterios de Landis y Koch en forma global (0: pobre; $0,01-0,02$ : leve; 0,21-0,40: regular; 0,41-0,60: moderado; 0,61-0,80: substancial y $0,81-1,00$ : casi perfecto) ${ }^{(14)}$ por sexo y edad categorizada ( $<13$ años, 14 a 15 años $y \geq 16$ años).

Realizamos un análisis multivariado a través de una regresión logística no condicional para identificar si alguna de las variables recolectadas (edad, peso, talla, índice de masa corporal, porcentaje de grasa, perímetro de cintura y cadera, y relación cintura-cadera) se comportaba como un factor de riesgo asociado al desacuerdo entre los adolescentes y el médico entrenado. La medida de asociación utilizada fue el riesgo relativo. Consideramos las siguientes variables como posibles confusoras: edad, talla, y peso. El nivel de significancia del estudio fue del $5 \%$. Para los análisis se utilizó el software STATA VE 12.0 (StataCorp. 2011. Stata Statistical Software: Release 12. College Station, TX: StataCorp LP).

\section{RESULTADOS}

Se incluyeron 229 participantes entre los 12 y 17 años (Tabla 1). El $51 \%$ de los participantes tenían entre 14 y 15 años. El índice de masa corporal (IMC) promedio de las mujeres fue de $20,5 \mathrm{Kg} / \mathrm{m}^{2}$ y el de los varones $19,4 \mathrm{Kg} / \mathrm{m}^{2}$. La concordancia entre la autoevaluación y la evaluación clínica de la escala de Tanner presentó un kappa ponderado $\left(\mathrm{kappa}_{\text {pond }}\right)$ de 0,554 con un acuerdo esperado de 73,8\% y un acuerdo obtenido de $88,3 \%$.

En la Tabla 2 se evidencia el número absoluto y el porcentaje de concordancia entre la evaluación clínica y la autoevaluación diferenciados por sexo. La autoevaluación en mujeres demostró una concordancia buena con respecto a la evaluación clínica $\left(k^{2}\right.$ ppa $\left._{\text {pond }}=0,6528\right)$. El $100 \%(n=119)$ de las mujeres determinaron correctamente el inicio de su desarrollo puberal (Tanner $\geq 2$ ), y el $68,9 \%$ $(n=82)$ de las mujeres clasificaron adecuadamente su estadio Tanner. Más mujeres subestimaron $(16,8 \%$, 
Tabla 1. Características demográficas y antropométricas de la población

\begin{tabular}{|c|c|c|c|c|}
\hline Características* & Total $(n=229)$ & Mujeres $(n=119)$ & Varones $(n=110)$ & Valor de $p^{\dagger}$ \\
\hline Edad (Años) & $14,6(1,3)$ & $14,4(1,3)$ & $14,8(1,34)$ & 0,02 \\
\hline Peso $(\mathrm{Kg})$ & $50,4(10,1)$ & $49,9(10,1)$ & $51,0(10,1)$ & 0,27 \\
\hline Talla (cm) & $158,4(8,86)$ & $155,8(5,7)$ & $161,3(10,6)$ & $<0,001$ \\
\hline IMC $\left(\mathrm{Kg} / \mathrm{m}^{2}\right)$ & $20,0(3,2)$ & $20,5(3,5)$ & $19,4(2,7)$ & 0,03 \\
\hline$\%$ de grasa & $19,8(8,2)$ & $24,8(6,5)$ & $14,4(6,2)$ & $<0,001$ \\
\hline Perímetro de cintura $(\mathrm{cm})$ & $69,6(10,6)$ & $69,2(12,2)$ & $70,1(8,4)$ & 0,09 \\
\hline Perímetro de cadera $(\mathrm{cm})$ & $86,5(8,7)$ & $87,7(9,4)$ & $85,2(7,8)$ & 0,07 \\
\hline Relación cintura-cadera & $0,8(0,1)$ & $0,8(0,1)$ & $0,8(0,1)$ & $<0,001$ \\
\hline
\end{tabular}

* Media (desviación estándar), ${ }^{\dagger}$ Prueba t de Student, IMC: índice de masa corporal

$\mathrm{n}=20)$ que sobreestimaron $(14,2 \%, \mathrm{n}=17)$ su desarrollo puberal por uno o dos estadios. Entre los acuerdos, la concordancia más frecuente se dio en el estadio 3 , con un porcentaje de $41,4 \%(n=34)$. No encontramos diferencias significativas entre el grupo de mujeres que concordó y el grupo que no concordó con el estadio de Tanner asignado por el médico entrenado. La autoevaluación en varones demostró una concordancia moderada con respecto a la evaluación clínica $\left(\mathrm{kappa}_{\text {pond }}=0,4638\right)$. El 93,6\% $(\mathrm{n}=103)$

Tabla 2. Evaluación clínica versus autoevaluación de la escala de Tanner en mujeres y varones adolescentes

\begin{tabular}{lccccc}
\hline $\begin{array}{l}\text { Evaluación } \\
\text { clínica }\end{array}$ & \multicolumn{5}{c}{ Autoevaluación * } \\
\hline Mujeres & T1 & T2 & T3 & T4 & T5 \\
\hline T1 & 2 & 0 & 0 & 0 & 0 \\
& $(1,68)$ & $(0,0)$ & $(0,0)$ & $(0,0)$ & $(0,0)$ \\
T2 & 1 & 7 & 2 & 0 & 0 \\
& $(0,84)$ & $(5,88)$ & $(1,68)$ & $(0,0)$ & $(0,0)$ \\
T3 & 0 & 2 & 34 & 14 & 0 \\
& $(0,0)$ & $(1,68)$ & $(28,5)$ & $(11,8)$ & $(0,0)$ \\
T4 & 0 & 1 & 8 & 31 & 1 \\
& $(0,0)$ & $(0,84)$ & $(6,72)$ & $(26,0)$ & $(0,84)$ \\
T5 & 0 & 0 & 0 & 8 & 8 \\
Varones & $(0,0)$ & $(0,0)$ & $(0,0)$ & $(6,72)$ & $(6,72)$ \\
T1 & & & & & \\
& $(1,81)$ & $(2,72)$ & $(0,91)$ & $(2,72)$ & $(0,0)$ \\
T2 & 1 & 10 & 9 & 0 & 0 \\
& $(0,91)$ & $(9,09)$ & $(8,18)$ & $(0,0)$ & $(0,0)$ \\
T3 & 1 & 3 & 13 & 6 & 0 \\
& $(0,91)$ & $(2,72)$ & $(11,8)$ & $(5,45)$ & $(0,0)$ \\
T4 & 0 & 4 & 10 & 25 & 4 \\
& $(0,0)$ & $(3,64)$ & $(9,09)$ & $(22,7)$ & $(3,64)$ \\
T5 & 0 & 0 & 1 & 10 & 4 \\
& $(0,0)$ & $(0,0)$ & $(0,91)$ & $(9,09)$ & $(3,64)$ \\
\hline
\end{tabular}

En blanco se encuentran los que clasificaron adecuadamente su estadio de Tanner, en gris oscuro se encuentran los que sobreestiman su estadio de Tanner y en gris claro se encuentran los que subestiman su estadio de Tanner

* $\mathrm{n}$ (\%), T1: estadio de Tanner 1, T2: estadio de Tanner 2, T3: estadio de Tanner 3, T4: estadio de Tanner 4, T5: estadio de Tanner 5 de los varones determinaron correctamente el inicio de su desarrollo puberal (Tanner $\geq 2)$, y el $49,0 \% \quad(n=54)$ de los varones clasificaron adecuadamente su estadio de Tanner. Más varones subestimaron $(27,2 \%, n=30)$ que sobreestimaron $(23,6 \%, n=26)$ su desarrollo puberal por uno o dos estadios. Entre los acuerdos, la concordancia más elevada fue observada en el estadio 4 , con un porcentaje de $46,2 \%(n=25)$. No encontramos diferencias significativas entre el grupo de adolescentes que concordó y el grupo que no concordó con el estadio de Tanner asignado por el médico entrenado.

La concordancia entre la autoevaluación y la evaluación clínica del desarrollo puberal disminuyó a medida que aumenta la edad (Tabla 3). Sin embargo, al realizar el análisis multivariado (no mostrado en la tabla) la única variable con una influencia significativa sobre la capacidad de acuerdo fue el sexo. La probabilidad de desacuerdo en la interpretación de la escala de Tanner en los varones, con el médico fue $63 \%$ mayor que en las mujeres, independiente de la edad, talla y peso (IC 95\%, 1,18-2,26).

\section{DISCUSIÓN}

En nuestro estudio, la concordancia general entre el examen clínico realizado por un médico y la autoevaluación realizado por los menores de edad fue moderada. Las mujeres tuvieron una concordancia buena comparada con los varones, quienes obtuvieron una concordancia moderada.

La concordancia y validez de la aplicación de la escala de Tanner en niños y adolescentes por autoevaluación o la evaluación por parte de las madres han arrojado resultados variables $(15,16)$, con algunos estudios reportando una concordancia interobservador adecuada ${ }^{(15-23)}$, mientras otros no recomiendan la autoevaluación como una medida confiable para la determinación del desarrollo puberal ${ }^{(11,15,24)}$, o reconocen sus limitaciones dependiendo de la variable a identificar, el sexo o el estado puberal ${ }^{(23,27)}$.

Algunos estudios han reportado niveles de acuerdo similares; sin embargo, son difícilmente comparables con nuestros resultados debido a que evaluaron por 
Tabla 3. Concordancia entre la evaluación clínica y la autoevaluación, según el sexo y la edad

\begin{tabular}{lccc}
\hline Característica & Acuerdo \% & $\begin{array}{c}\text { Acuerdo } \\
\text { esperado } \%\end{array}$ & k (DE) \\
\hline Sexo & & & \\
$\quad$ Mujeres & 92,0 & 77,0 & $0,653(0,060)$ \\
$\quad$ Varones & 84,3 & 70,7 & $0,464(0,062)$ \\
Edad (años) & & & \\
$\quad<13$ & 90,2 & 72,9 & $0,640(0,089)$ \\
$14-15$ & 82,2 & 76,9 & $0,491(0,061)$ \\
$>16$ & 86,6 & 78,8 & $0,391(0,086)$ \\
\hline
\end{tabular}

k: Coeficiente kappa de Cohen

DE: desviación estándar

separado las características genitales y las del vello púbico. Desmangles, et al. ${ }^{(15)}$, encontraron que el nivel de acuerdo de la autoclasificación de Tanner para vello púbico fue bueno en las mujeres (kappa=0,68). Sin embargo, al analizar la autoevaluación del desarrollo mamario el nivel de acuerdo fue moderado (kappa=0,49). En el caso de los varones, realizaron la escala de Tanner conjunta (vello púbico y desarrollo genital), y encontraron un nivel de acuerdo moderado (kappa=0,49). Rasmussen, et al. ${ }^{(25)}$, evaluaron 898 niños daneses de 7,4 a 14,9 años, encontrando un nivel de acuerdo débil (kappa=0,28 vs. kappa=0,33) en el desarrollo mamario y genital, y un nivel de acuerdo moderado (kappa=0,55 vs. kappa=46) en el desarrollo de vello púbico en las niñas y niños que autoevaluaron su estadio de Tanner.

Además, los niveles de acuerdo tienden a ser menores en la autoevaluación del desarrollo genital, tanto para mujeres como para varones al ser comparados con los niveles de acuerdo del desarrollo del vello púbico, lo que sugiere que la verdadera dificultad de la autoclasificación del desarrollo puberal se encuentra en la determinación del desarrollo de las glándulas mamarias para las mujeres, y de los testículos y el pene para los varones. Rollof, et al (21), utilizaron un orquidómetro como herramienta para mejorar la utilidad de la autoevaluación del desarrollo puberal en varones de 10 a 16 años. A pesar de reportarlo como un método útil, el porcentaje de acuerdo fue de $36 \%$. Nuestros resultados demuestran, además, que el sexo masculino tiene mayor riesgo para la autoclasificación errónea del estadio de desarrollo puberal, independiente de la edad y el IMC. No encontramos una asociación entre la edad, la talla, el peso o el IMC, a pesar de que se han reportado estas características como posibles factores influenciadores.

Tanto mujeres como varones tendieron a subestimar su desarrollo puberal; sin embargo, una mayor proporción de varones sobreestimaron su estadio con respecto a mujeres, resultados similares a los reportados en otras poblaciones ${ }^{(15,27)}$, lo que podría sugerir que, en nuestra sociedad, al igual que en otras, la maduración temprana es socialmente deseable para los varones y no tan deseada por las mujeres durante la adolescencia.
El estudio SIMAC es el primer estudio en Colombia en evaluar la concordancia de la autoevaluación del desarrollo puberal mediante la escala de Tanner de los adolescentes, con la evaluación efectuada por un médico entrenado, demostrando que la autoevaluación del desarrollo puberal es una medida inexacta, y que ser varón aumenta la probabilidad de desacuerdo, independiente de la edad, talla y peso. No consideramos el estrato socioeconómico o el nivel educativo, debido a que la muestra fue extraída de un mismo entorno social y educativo.

Nuestro estudio posee ciertas limitaciones, tales como la edad de inclusión de los participantes lo que limitó la posibilidad de analizar la sensibilidad y la especificidad de la autoevaluación, así como la confiabilidad de la estimación realizada, debido a que el número de adolescentes clasificados como Tanner 1 fue pequeño. Adicionalmente, no dividimos la escala de Tanner por desarrollo mamario, genital masculino y vello púbico, situación que limita la posibilidad de comparación con otros estudios, y a su vez su uso podría haber mejorado la autoevaluación. Por último, a pesar de no haber encontrado una asociación con el IMC, reconocemos que el hecho de tener participantes en peso normal limita el poder estadístico para detectar este tipo de influencias. Teniendo en cuenta que, un IMC corporal aumentado, sobre todo en mujeres, podría inducir a una clasificación errónea del estadio del desarrollo mamario. Al no tener una muestra representativa, nuestros resultados no pueden ser generalizados, por lo cual se requieren de más estudios en relación con este tema, reconociendo el impacto en el diagnóstico de alteraciones nutricionales, endocrinas o metabólicas, que podría llegar a tener la autoevaluación del desarrollo sexual. A pesar de esto, nuestro estudio demuestra la limitada utilidad de la autoevaluación para la estadificación del desarrollo puberal para población infantil y adolescente colombiana.

Los estudios demográficos de los últimos 15 a 20 años han evidenciado una tendencia hacia la pubertad temprana en las mujeres, por lo tanto, como fue mencionado anteriormente, es indispensable actualizar de forma periódica y regional los límites de edad para un desarrollo puberal normal. Adicionalmente, teniendo en cuenta que la escala de Tanner es la herramienta utilizada por convención para el diagnóstico de retraso puberal o pubertad precoz, es necesario fomentar y ampliar la realización de estudios e investigaciones en este campo.

En conclusión, la autoevaluación de la maduración sexual en la población estudiada no fue lo suficientemente precisa para reemplazar el examen médico, sobre todo en el sexo masculino. Sería necesario realizar más estudios en relación con este tema, reconociendo el impacto que podría llegar a tener la autoevaluación del desarrollo sexual.

Agradecimientos: Agradecemos al Colegio Centro de Comercio (CEDECO) por su participación y promoción de las actividades. 
Contribuciones de autoría: De acuerdo con los cuatro criterios de autoría, todos los autores realizaron contribuciones significativas a la concepción y diseño del manuscrito. PAC y JO se encargaron del análisis e interpretación de los datos. SMRQ, MAAA y AMA realizaron la redacción del contenido del artículo, la cual fue revisada de forma crítica, corregida y aprobada por JO, DC, PAC y PLJ. Todos los autores asumen la responsabilidad frente a todos los aspectos del manuscrito, para garantizar que los asuntos relativos a la exactitud o integridad de cualquier parte del mismo serán adecuadamente investigados y resueltos.

Financiamiento: Estudio cofinanciado por COLCIENCIAS (Fuerza muscular y capacidad aeróbica relación simbiótica en escolares con bajo peso al nacer y riesgo metabólico-Estudio SIMAC. Contrato $\mathrm{N}^{\circ}$. 645-2014. Código $\mathrm{N}^{\circ}$. 651765741093) y desarrollado en el Colegio Centro de Comercio (CEDECO) de Piedecuesta.

Conflicto de intereses: Ninguno de los autores tiene intereses en conflicto en el manuscrito.

\section{REFERENCIAS BIBLIOGRÁFICAS}

1. Marchant C, Bancalari R, Díaz C, Zamorano J, Cerda V, Fernández M, et al. La autoevaluación es un método poco confiable para establecer el desarrollo puberal en escolares. Rev Chil Pediatr. 2012;83(4):345-51. doi: 10.4067/S037041062012000400004 .

2. Biro FM, Chan YM. Normal puberty [Internet].UptoDate; 2017 [consultado el2 de agosto de 2017]. Disponible en: https:// www.uptodate.com.aure.unab.edu.co/ contents/normalpuberty?source=search result \& s e a r c h $=$ p uberty \&selectedTitle $=1 \sim 150$

3. Susman EJ, Houts RM, Steinberg L, Belsky J, Cuaffman E, Dehart G, et al. Longitudinal development of secondary sexual characteristics in girls and boys between ages $91 / 2$ and 151/2 years. Arch Pediatr Adolesc Med. 2010;164(2):16673. doi: 10.1001/archpediatrics.2009.261.

4. Biro FM, Lucky AW, Huster GA, Morrison JA. Pubertal staging in boys. J Pediatr. 1995;127(1):100-2.

5. Tanner, JM. Growth at adolescence. 2nd ed. Springfield: Thomas; 1962.

6. Marshall WA, Tanner JM. Variations in pattern of pubertal changes in girls. Arch Dis Child. 1969;44(235):291-303.

7. Marshall WA, Tanner JM. Variations in the pattern of pubertal changes in boys. Arch Dis Child. 1970;45(239):13-23.

8. Lejarraga H. Maduración infantil, una aproximación pediátrica a algunos métodos para su evaluación. Rev Hosp Niños (Buenos A). 1976;18:69-78.

9. Temboury Molina, MC. Desarrollo puberal normal: Pubertad precoz. Rev Pediatr Aten Primaria. 2009;11(Supl.16):127-42.

10. Raman A, Lustig R, Fitch M, Fleming S. Accuracy of self-assessed tanner staging against hormonal assessment of sexual maturation in overweight AfricanAmerican Children. J Pediatr Endocrinol Metab. 2009;22(7):609-22.
11. Azevedo JCV, Brasil LMP, Macedo TBMA, Pedrosa LFC, Arrais RF. Comparison between objective assessment and self-assessment of sexual maturation in children and adolescents. J Pediatr (Rio J). 2009;85(2):132-42 doi: 10.1590/S002175572009000200009 .

12. Marshall WA, Tanner JM. Variations in pattern of pubertal changes in girls. Arch Dis Child.1969;44:291-303.

13. Marshall WA, Tanner JM. Variations in the pattern of pubertal changes in boys. Arch Dis Child. 1970;45:13-23.

14. Landis LR, Koch GG. The measurement of observer agreement for categorical data. Biometrics. 1977;33 (1):159-77.

15. Desmangles JC, Lappe JM, Lipaczewski G, Haynatzki G. Accuracy of pubertal Tanner staging self-reporting. J Pediatr Endocrinol Metab. 2006;19(3):213-21.

16. Terry MB, Goldberg M, Schechter $S$, Houghton LC, White ML, O’Toole K et al. Comparison of clinical, maternal, and self-pubertal assessments: implications for health studies. Pediatrics. 2016;138(1). doi: 10.1542/peds.2015-4571.

17. Taylor SJ, Whincup PH, Hindmarsh PC, Lampe F, Odoki K, Cook DG. Performance of a new pubertal selfassessment questionnaire: a preliminary study. Paediatr Perinat Epidemiol. 2001;15(19):88-94.

18. Wacharasindhu S, Pri-Ngam P, Kongchonrak T. Self-assessment of sexual maturation in Thai children by Tanner photograph. J Med Assoc Thai. 2002;85(3):308-19.

19. Chan N, Sung RY, Kong AP, Goggins WB, So HK, Nelson EA. Reliability of pubertal self-assessment in Hong Kong Chinese children.J Paediatr Child Health. 2008;44(6):353-8 doi: 10.1111/j.14401754.2008.01311.x.

20. Rollof L, Elfving M. Evaluation of selfassessment of pubertal maturation in boys and girls using drawings and orchidometer. J Pediatr Endocrinol Metab. 2012;25(12):125-9.
21. Sun Y, Tao FB, Su PY. Self-assessment of pubertal Tanner stage by realistic colour images in representative Chinese obese and non-obese children and adolescents. Acta Paediatr. 2012;101(4):163-6 doi: 10.1111/j.1651-2227.2011.02568.x.

22. Jaruratanasirikul S, Kreetapirom P, Tassanakijpanich $\mathrm{N}$, Sriplung $\mathrm{H}$. Reliability of pubertal maturation selfassessment in a school-based survey. J Pediatr Endocrinol Metab. 2015;28(34):367-74 doi: 10.1515/jpem-2014-0053.

23. Lee K, Valeria B, Kochman C, Lenders CM. Self-assessment of height, weight, and sexual maturation: validity in overweight children and adolescents. J Adolesc Health. 2006;39(3):346-52.

24. Rasmussen AR, Wohlfahrt-Veje C, de Renzy-Martin KT, Hagen CP, Tinggaard J, Mouritsen A et al. Validity of self-assessment of pubertal maturation. Pediatrics. 2015;135(1):86-93 doi: 10.1542/ peds.2014-0793.

25. Taylor SJ, Whincup PH, Hindmarsh PC, Lampe F, Odoki K, Cook DG. Performance of a new pubertal selfassessment questionnaire: a preliminary study. Paediatr Perinat Epidemiol. 2001;15(1):88-94.

26. Rabbani A, Noorian $S$, Fallah JS, Setoudeh A, Sayarifard F, Abbasi F. Reliability of pubertal self-assessment method: an Iranian study. Iran J Pediatr. 2013;23(3):327-32.

27. Schlossberger NM, Turner RA, Irwin CE. Validity of self-report of pubertal maturation in early adolescents. J Adolesc Health. 1992;13(2):109-13.

Correspondencia: Prof. Patricio LopezJaramillo $M D, P h D, F A C P$

Dirección: Fundación Oftalmológicade Santander (FOSCAL/FOSCAL Internacional). Calle 158 \# 28a - 95, local 101-102. Urbanización El Bosque. Floridablanca, Santander, Colombia Teléfono: (57) 76797979 Ext: 6163 - 6162 Correo electrónico:jplopezj@gmail.com 